\title{
Temperature probes in binary granular gases
}

\author{
Alain Barrat ${ }^{1}$, Vittorio Loreto ${ }^{2}$ and Andrea Puglisi ${ }^{2}$ \\ 1 Laboratoire de Physique Théorique Unité Mixte de Recherche UMR 8627, \\ Bâtiment 210, Université de Paris-Sud, 91405 Orsay Cedex, France \\ 2 "La Sapienza" University, Physics Department and INFM-SMC, \\ Unità di Roma1, P.le A. Moro 2, 00185 Rome, Italy
}

(Dated: June 4, 2018)

\begin{abstract}
We investigate the validity of Fluctuation-Dissipation (FD) relations for a mixture of two granular gases with different physical properties (restitution coefficients or masses) subject to stochastic driving. It is well known that the partial granular temperatures $T_{1}$ and $T_{2}$ of the two components are different, i.e. energy equipartition is broken. We observe, with numerical simulations of inelastic hard disks in homogeneous and non-homogeneous situations, that the classical equilibrium GreenKubo relations are satisfied separately for each component of the gas, the role of the equilibrium temperature being played by the granular temperature of each component. Of particular interest is the limit in which one of the two components consists of only one particle, representing a nonperturbing thermometer. In this case it turns out that such a thermometer is measuring its own temperature and not that of the surrounding granular media, which in general will be different.
\end{abstract}

PACS numbers:

\section{INTRODUCTION}

Granular gases 1], i.e. gases of particles interacting through dissipative collisions, represent an important paradigm for the study of non-equilibrium stationary states. Due to the dissipative nature of the interactions, granular gases have to be considered as open systems and therefore concepts from equilibrium thermodynamics cannot be applied, at least in a straightforward way. However, by analogy with molecular gases, a "granular temperature" $T_{g}$ can be defined in terms of the kinetic energy per particle. If the system is driven by an external energy input a stationary state is reached: $T_{g}$ fluctuates around a given value, while a flux of energy from the driving source goes into the system and is dissipated through inelastic collisions. Experiments, theory and simulations have recently shown that in a mixture of different grains, as soon as the inter-particle collisions dissipate energy, the equipartition of energy among the mixture components is lost 2, 3, 4, 5, 6, 7, 8, 9, 10, 11, 12 even in the tracer limit [13]. Although in sharp contrast with the behaviour of molecular gases at equilibrium, this violation is not surprising because in a generic open system the equipartition of energy is not expected. However, it is natural to ask whether $T_{g}$ could have some different "equilibrium" meaning, or if it is just a measure of velocity fluctuations. A hallmark of equilibrium phenomena is the well-known fluctuation dissipation theorem, relating the response of a system to a perturbation to the corresponding correlation function measured in the unperturbed system: the response of an observable $B$ at time $t$ to an impulsive perturbation $h_{A}$ at time $t=0$ can be obtained as

$$
\frac{\delta\langle B\rangle}{\delta h_{A}}=-\frac{1}{T} \frac{\partial}{\partial t}\langle B(t) A(0)\rangle
$$

where $A$ is the observable conjugated to $h_{A}$ and $T$ is the equilibrium temperature of the system (brackets denote averaging over thermal history). A recent investigation has shown that, for non-equilibrium monodisperse driven granular gases, this relation is still obeyed if the equilibrium temperature is replaced by the granular temperature [14].

A natural question then arises: what happens to the relation (11) in a mixture which displays more than one granular temperature? In this Letter, we will address this question in the case of a binary mixture subject to a homogeneous driving. It turns out that each species obeys an FDT relation with its own granular temperature [15] as proportionality factor. This result is particularly relevant to experiments where a tracer, immersed in the granular gas and acting as a probe (and in particular as a thermometer), may have different physical properties with respect to the surrounding gas [17]. In equilibrium measurements, the temperature does not depend on the thermometer. For granular materials, instead, the thermometer measures its own temperature, in general different from that of the surrounding gas.

\section{MODEL}

We consider a volume $V$ containing a mixture of $N=$ $N_{1}+N_{2}$ inelastic hard spheres (IHS) in dimension $d=2$, $N_{1}$ and $N_{2}$ being the number of particles in component 1 and 2 of the mixture, respectively. The spheres have diameters $\sigma$ (identical for the two species) and masses $m_{s_{i}}$ (where $1 \leq i \leq N$ and $s_{i}$ is the species index, 1 or 2 , of particle $i$ ). In a collision between spheres $i$ and $j$, characterized by the inelasticity parameter called coefficient of normal restitution $\alpha_{s_{i} s_{j}}$, the pre-collisional velocity of particle $i, \boldsymbol{v}_{i}$, is transformed into the post-collisional ve- 
locity $\boldsymbol{v}_{i}^{\prime}$ such that

$$
\boldsymbol{v}_{i}^{\prime}=\boldsymbol{v}_{i}-\frac{m_{s_{j}}}{m_{s_{i}}+m_{s_{j}}}\left(1+\alpha_{s_{i} s_{j}}\right)\left(\widehat{\boldsymbol{\sigma}} \cdot \boldsymbol{v}_{i j}\right) \widehat{\boldsymbol{\sigma}}
$$

where $\boldsymbol{v}_{i j}=\boldsymbol{v}_{i}-\boldsymbol{v}_{j}$ and $\widehat{\boldsymbol{\sigma}}$ is the center to center unit vector from particle $i$ to $j\left(\alpha_{s_{i} s_{j}}=\alpha_{s_{j} s_{i}}\right.$ so that the total linear momentum $m_{s_{i}} \boldsymbol{v}_{i}+m_{s_{j}} \boldsymbol{v}_{j}$ is conserved). The granular temperature of species $s$ is given by its mean kinetic energy $T_{s}=\left\langle m_{s} v_{s}^{2}\right\rangle / d$.

The loss of energy due to collisions can be compensated in various ways. In experiments the energy is typically supplied at the boundaries, leading the system to a heterogeneous stationary state [3, 4, 5, 18]. In order to avoid the complication of strong temperature heterogeneities, we will use a homogeneous driving in the form of a "thermostat": in this mechanism (which recently has attracted the attention of many theorists 19, 20, 21, 22, 23, 24, 25]), the particles are submitted, between collisions, to a random force in the form of an uncorrelated white noise (e.g. Gaussian) with the possible addition of a viscous term. The equation of motion for a particle is then

$$
m_{i} \frac{d \boldsymbol{v}_{i}}{d t}=\boldsymbol{F}_{i}+m_{i} \boldsymbol{R}_{i}-\zeta \boldsymbol{v}_{i}
$$

where $\boldsymbol{F}_{i}$ is the force due to inelastic collisions, $\zeta$ is the viscosity coefficient and $\left\langle R_{i \alpha}(t) R_{j \beta}\left(t^{\prime}\right)\right\rangle=\xi_{0}^{2} \delta_{i j} \delta_{\alpha \beta} \delta(t-$ $\left.t^{\prime}\right)$, where Greek indexes refer to Cartesian coordinates [26].

At the level of Boltzmann kinetic equation, the temperature ratio of a binary granular mixture subject to stochastic driving of the form given above has been obtained in [7] for the case $\zeta=0$ and in [1] for $\zeta \neq 0$.

\section{METHODS}

We have used two different simulation methods: the Direct Simulation Monte Carlo (DSMC) 28, 29] which neglects pre-collisional correlations and therefore enforces the molecular chaos hypothesis (factorization of the twoparticles distribution functions) and Molecular Dynamics (MD) simulations. The DSMC algorithm can be used to simulate the homogeneous Enskog-Boltzmann equation, i.e. any particle can collide with any other in the whole volume $V$, or to simulate an a priori nonhomogeneous system, by dividing the space into small cells (of linear size smaller than the mean free path) and enforcing molecular chaos in every single cell. On the other hand, molecular dynamics (MD) simulations integrate the equations of motion of the IHS: in this case we consider $N$ hard spheres in a square box of linear size $L=\sqrt{V}$, with periodic boundary conditions, random initial velocities, and we use an event-driven algorithm to study their dynamics. All the investigation methods used have shown similar results. We have used sizes $N=500$ to $N=5000$, and averaged over up to 10000 realizations of the dynamics.

Our analysis relies on two sets of independent measurements, i.e. two choices of the pair response-correlation to be measured. The first one consists in a measure of mobility and diffusivity. The mean-square displacement (MSD) for each species,

$$
B_{1(2)}\left(t, t^{\prime}\right)=\frac{1}{d N_{1(2)}} \sum_{j=1}^{N_{1(2)}}\left\langle\left|\boldsymbol{r}_{j}(t)-\boldsymbol{r}_{j}\left(t^{\prime}\right)\right|^{2}\right\rangle,
$$

behaves as $\sim 4 D_{1(2)}\left(t-t^{\prime}\right)$ for large time differences. The mobility of a tracer particle is measured by applying a constant and small [27] drag force $\boldsymbol{\xi}=\xi \boldsymbol{e}_{x}$ to a given particle, labeled 0 , for times $t>t^{\prime}$. Due to the viscosity induced by the collisions with other particles, the perturbed particle will reach at large times a constant velocity $\mu$, related to the response by

$$
\chi_{1,2}\left(t, t^{\prime}\right)=\frac{1}{\xi}\left\langle\left(\boldsymbol{r}_{0}(t)-\boldsymbol{r}_{0}\left(t^{\prime}\right)\right) \cdot \boldsymbol{e}_{x}\right\rangle \approx \mu t \text { at large } \mathrm{t} .
$$

By successively using as test particle one particle of each species, one obtains the two responses $\chi_{1}$ and $\chi_{2}$, and thus the mobilities $\mu_{1}$ and $\mu_{2}$. Two Einstein relations $\left(\mu_{i}=2 D_{i} / T_{i}\right)$ can then be checked, e.g. by plotting $\chi_{i}$ vs. $B_{i}$.

Another totally independent way of checking FD relations in granular gases has also been used in [14]: once a steady-state has been reached, the system is perturbed impulsively at a given time $t_{0}$ by a non-conservative force applied (non-uniformly) on every particle (we will take $t_{0}=0$ without loss of generality). The response is then monitored at later times. The force acting on particle $i$ is

$$
\mathbf{F}\left(\mathbf{r}_{i}, t\right)=\gamma_{i} \boldsymbol{\xi}\left(\mathbf{r}_{i}, t\right)
$$

with the properties $\boldsymbol{\nabla} \times \boldsymbol{\xi} \neq 0, \boldsymbol{\nabla} \cdot \boldsymbol{\xi}=0$, where $\gamma_{i}$ is a particle dependent variable with randomly assigned \pm 1 values. A simple case is realized by a transverse perturbation $\boldsymbol{\xi}(\mathbf{r}, t)=\left(0, \xi \cos \left(k_{x} x\right) \delta\left(t-t_{0}\right)\right)$ [27], where $k_{x}$ is compatible with the periodic boundary conditions, i.e. $k_{x}=2 \pi n_{k} / L_{x}$ with $n_{k}$ integer and $L_{x}$ the linear horizontal box size. The staggered response function (i.e. the current induced at $t$ by the perturbation at $t_{0}$ ), and the conjugated correlation,

$$
\begin{aligned}
R\left(t, t_{0}\right) & =\frac{1}{\xi}\left\langle\sum_{i} \gamma_{i} \dot{y}_{i}(t) \cos \left(k_{x} x_{i}(t)\right)\right\rangle, \\
C\left(t, t_{0}\right) & =\left\langle\sum_{i} \dot{y}_{i}(t) \dot{y}_{i}\left(t_{0}\right) \cos \left\{k_{x}\left[x_{i}(t)-x_{i}\left(t_{0}\right)\right]\right\}\right\rangle
\end{aligned}
$$

are related, at equilibrium, to the correlation by the $\mathrm{FD}$ relation $R\left(t, t_{0}\right)=\frac{\beta}{2} C\left(t, t_{0}\right), T=1 / \beta$ being the equilibrium temperature. 
Ref. 14] has shown the validity of this relation in the context of a monodisperse granular media heated by a thermal bath with temperature $T_{b}$, reaching in this way a stationary state with granular temperature $T_{g}<T_{b}$. In this case the FD relation holds by replacing $T$ with $T_{g}$. For a binary mixture, two sets $\left(C_{i}, R_{i}\right)(i=1,2)$ of correlation and response are measured separately, thus obtaining two plots $C_{i}$ vs. $R_{i}$.

There are different reasons to test various pairs of response and correlation functions. First, at equilibrium FDT links any couple of conjugated correlation and response function with the same temperature. Out of equilibrium, it is possible, a priori, that FDT could be valid for some observables and not for others. Moreover, mean square displacement and mobility correspond to the exploration of the long time regime, while $C_{i}$ and $R_{i}$ decay quickly (a few collisions per particle) and therefore yield the short time behaviour. FDT could be valid in some time regimes and not in others. It must also be stressed that the measure of diffusion and mobility requires large times, so that averaging over many realizations becomes computationally very demanding and less precision is numerically available. In MD simulations in particular we have measured only the relation between $C_{i}$ and $R_{i}$.

\section{RESULTS}

In all the simulations performed (MD and DMSC) the values of the temperature ratios $T_{1} / T_{2}$ obtained are in good agreement with [7]. This is not surprising for homogeneous DSMC, while it is less obvious in MD (the agreement is mostly due to the low packing fraction $n$ used).

\section{Fluctuation-Dissipation ratio for the different components}

The measures of $B_{i}(t)$ and $\chi_{i}(t)$ allow us to check the linearity with $t$ at large times: $B_{i}(t) \approx 4 D_{i} t$ and $\chi_{i}(t) \approx \mu_{i} t$. Moreover, figures 1 and 2 clearly show that the Einstein relations are obeyed separately by the two components of the mixture, each with its own temperature: to the numerical accuracy one obtains $\chi_{i}(t)=$ $\frac{1}{2 T_{i}} B_{i}(t)$. Note that mobilities and diffusion coefficients of each species are different from their value in a monodisperse case. We have considered various values of inelasticities, mass ratios, number density ratios, and kinds of thermostat, obtaining that this result is robust with respect to all these variations. We also note that a recent study [16] has shown that the Einstein relation is not valid in its usual form in the case of an impurity immersed in an homogeneously cooling granular: the main reason is that extra terms arise due to the evolution of the granular temperature with time. Since we are here concerned with steady-states, these extra terms are not present in the case studied. The other source of deviations, namely the deviation from Gibbs state, leads to small deviations 16] that could be difficult to detect [15].
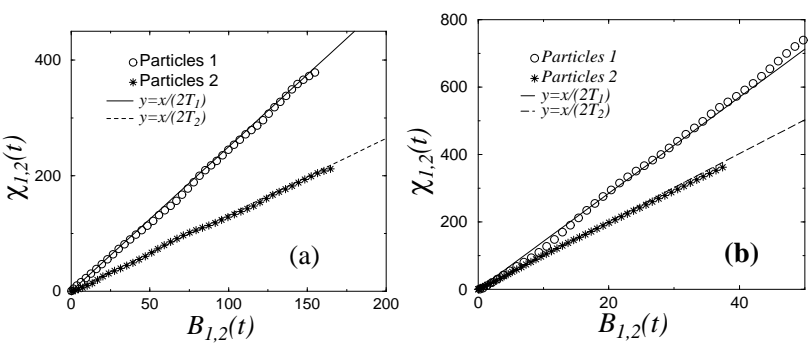

FIG. 1: Random force $(\zeta=0)$, homogeneous DSMC: mobilities $\chi_{1,2}$ vs. MSD $B_{1,2} ;(\mathrm{a}): \alpha_{11}=0.3, \alpha_{12}=0.5, \alpha_{22}=0.7$, $m_{2}=3 m_{1}, T_{1} \approx 0.2, T_{2} \approx 0.38 ;(\mathrm{b}): \alpha_{11}=\alpha_{12}=\alpha_{22}=0.9$, $m_{2}=5 m_{1}, T_{1} \approx 0.035, T_{2} \approx 0.05$. Symbols are numerical data, lines have slope $1 /\left(2 T_{1}\right)$ and $1 /\left(2 T_{2}\right)$.
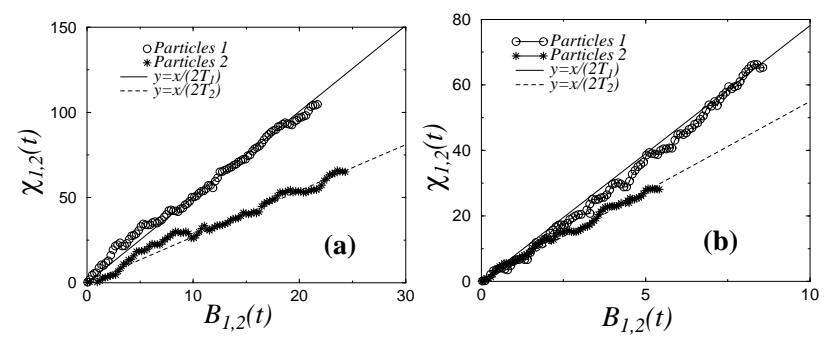

FIG. 2: Random force $(\zeta=0)$, DSMC with cells: mobilities $\chi_{1,2}$ vs. $\operatorname{MSD} B_{1,2}$. (a): $\alpha_{11}=0.3, \alpha_{12}=0.5, \alpha_{22}=0.7$, $m_{2}=3 m_{1}, T_{1} \approx 0.1, T_{2} \approx 0.185 ;(\mathrm{b}): \alpha_{11}=\alpha_{12}=\alpha_{22}=0.9$, $m_{2}=5 m_{1}, T_{1} \approx 0.064, T_{2} \approx 0.09$. Symbols are numerical data, lines have slope $1 /\left(2 T_{1}\right)$ and $1 /\left(2 T_{2}\right)$.

We now turn to the measure of $R_{i}(t)$ and $C_{i}(t)$. Technical details of the numerical procedure to perform this measure are given in [14]. From the definitions of $\boldsymbol{\xi}, C_{i}$ and $R_{i}$, it is clear that $R_{i}(0)=1 /\left(2 m_{i}\right)$, and $C_{i}(0)=$ $\left\langle v_{i}^{2}\right\rangle=T_{i} / m_{i}$. On the other hand, $\lim _{t \rightarrow \infty} R_{i}(t)=$ $\lim _{t \rightarrow \infty} C_{i}(t)=0$. Thus we have plotted in figure 3 the functions $2 m_{i} R_{i}(t)$ vs. $m_{i} C_{i}(t)$. The FD relation $R_{i}(t)=\frac{1}{2 T} C_{i}(t)$ is verified replacing $T$ by the partial granular temperature $T_{1}$ and $T_{2}$ of each component. The same result holds for different $n_{k}\left(n_{k} \neq 0\right.$ in order to satisfy the properties of the perturbing force (6)).

The MD simulations (whose results are reported in figure 31) are more realistic since they include excluded volume effects and collision-induced correlations (which break the Molecular Chaos hypothesis, see for example 22]). However, at not too high packing fractions, we still observe the same results for FD relations. Larger packing fractions lead to strong heterogeneities in both density and granular temperature, giving rise to deviations from FD [15]. 


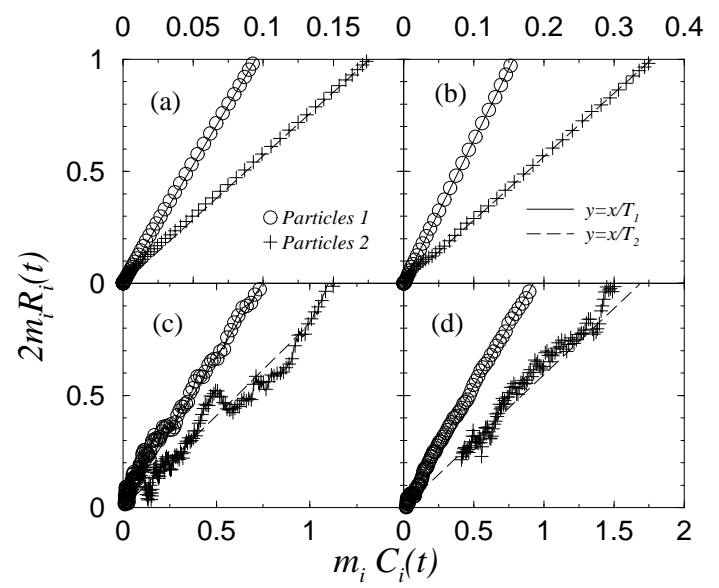

FIG. 3: Random force (i.e. $\zeta=0$ ), DSMC with cells, and MD: $2 m_{i} R_{i}(t)$ vs. $m_{i} C_{i}(t)(i=1,2)$. Here $n_{k}=4 . C_{i}(0)=T_{i} / m_{i}$, $R_{i}(0)=1 /\left(2 m_{i}\right)$. In all cases the circles correspond to the FD plot for the type 1 particles, the pluses to the FD plot for type 2 particles. Straight lines have slope $1 / T_{1}$, dashed lines have slope $1 / T_{2}$. Top: DSMC simulations. (a): $\alpha_{11}=0.3$, $\alpha_{12}=0.5, \alpha_{22}=0.7, m_{2}=3 m_{1}, T_{1} \approx 0.09, T_{2} \approx 0.18 ;(\mathrm{b})$ : $\alpha_{11}=\alpha_{12}=\alpha_{22}=0.7, m_{2}=5 m_{1}, T_{1} \approx 0.16, T_{2} \approx 0.35 ;$ Bottom: MD simulations, with $n=0.1$. (c): $N_{1}=N_{2}$, $\alpha_{11}=0.7, \alpha_{12}=0.8, \alpha_{22}=0.9, m_{2}=3 m_{1}, T_{1} \approx 0.75$, $T_{2} \approx 1.22 ;(\mathrm{d}): N_{1}=9 N_{2} ; \alpha_{11}=\alpha_{12}=\alpha_{22}=0.9, m_{2}=5 m_{1}$, $T_{1} \approx 0.93, T_{2} \approx 1.69$.

\section{Fluctuation-Dissipation ratio for the whole system}

An interesting question concerns what happens to the Fluctuation-Dissipation ratio when measured for the whole system and not separately for the different components of the mixture. In other words one could ask whether one can define an effective temperature for the whole system and what is the relation of this temperature with the temperatures defined above for the two components of the mixture, or with the global temperature $T=x_{1} T_{1}+x_{2} T_{2}$ (where $\left.x_{1,2}=\frac{N_{1,2}}{N}\right)$.

A global measure would give, for the mean-square displacement

$$
B(t)=x_{1} B_{1}(t)+x_{2} B_{2}(t)
$$

and for the response

$$
\chi(t)=x_{1} \chi_{1}(t)+x_{2} \chi_{2}(t) .
$$

Using the previously checked result that $\chi_{i}(t) \approx \mu_{i} t$ and $B_{i}(t) \approx 4 D_{i} t$, one obtains that $B(t) \approx 4 D t$ and $\chi(t) \approx \mu t$, with $D=x_{1} D_{1}+x_{2} D_{2}$ and $\mu=x_{1} \mu_{1}+x_{2} \mu_{2}$. The Einstein relation for the global case thus reads:

$$
\frac{2 D}{\mu}=\frac{\left(x_{1} D_{1}+x_{2} D_{2}\right) T_{1} T_{2}}{x_{1} D_{1} T_{2}+x_{2} D_{2} T_{1}}
$$

It is clear that the ratio $\frac{2 D}{\mu}$ corresponds to the global granular temperature only when $T_{1}=T_{2}$, i.e. when equipartition is satisfied. In figure 4 we present evidences supporting this view.

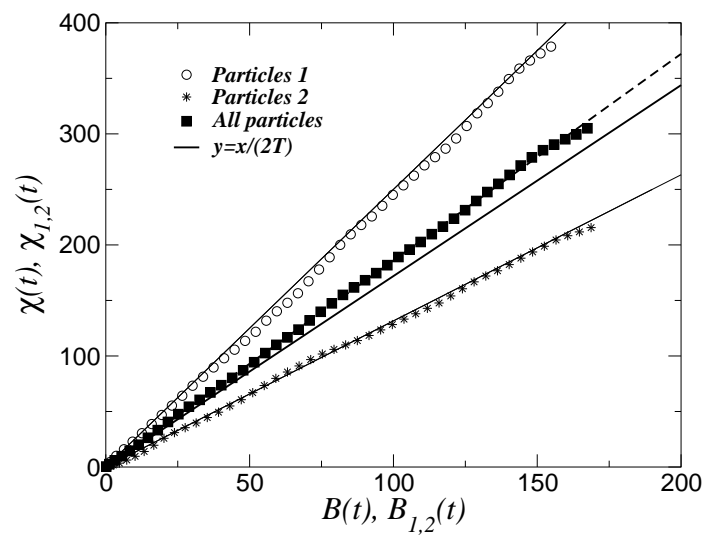

FIG. 4: Homogeneous DSMC, random force, $\alpha_{11}=0.3, \alpha_{12}=$ $0.5, \alpha_{22}=0.7, m_{2}=3 m_{1}, N_{1}=N_{2}$. The filled squares correspond to the numerical data of $\chi(t)$ vs. $B(t)$. Circles and stars correspond respectively to particles of type 1 and 2 , and the thin lines have slopes $1 /\left(2 T_{1}\right)$ and $1 /\left(2 T_{2}\right)$. The thick continuous line has slope $1 /(2 T)$ while the dashed line has slope $\mu /(4 D)$ with $2 D / \mu$ given by eq. 9 Here $T \approx 0.29$ while $2 D / \mu \approx 0.27$.

Another way to consider the problem is to look at the ratio between $R(t)=x_{1} R_{1}(t)+x_{2} R_{2}(t)$ and $C(t)=$ $x_{1} C_{1}(t)+x_{2} C_{2}(t)$. In this case the impossibility to define an effective temperature reflects itself in the non-constant ratio between $C(t)$ and $R(t)$, as we show in figure 5 .

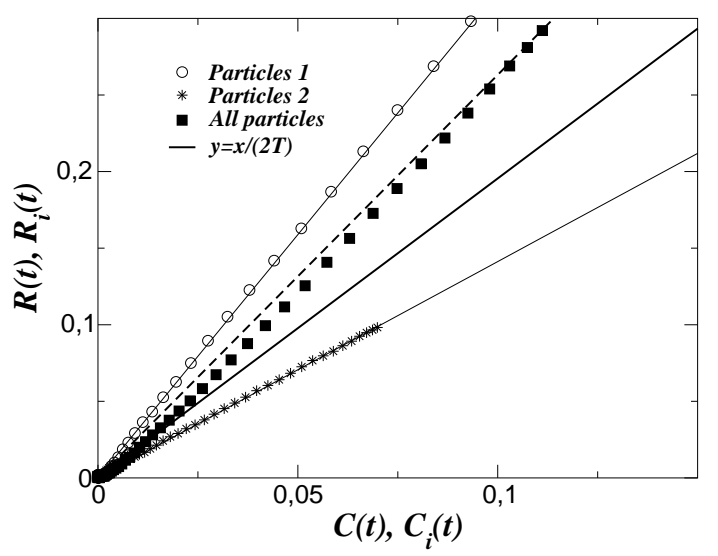

FIG. 5: DSMC with cells, random force, $\alpha_{11}=\alpha_{12}=\alpha_{22}=$ $0.7, m_{2}=5 m_{1}, N_{1}=N_{2}$. The filled squares correspond to the numerical data of $R(t)$ vs. $C(t)$. Circles and stars correspond respectively to $R_{1}(t)$ vs. $C_{1}(t)$ and $R_{2}(t)$ vs. $C_{2}(t)$. Thin lines have slopes $1 /\left(2 T_{1}\right)$ and $1 /\left(2 T_{2}\right)$. The thick continuous line has slope $1 /(2 T)$; the dashed line is a guide to the eye showing that $R(t)$ vs. $C(t)$ is not a straight line.

It should be remarked how these properties of the global Fluctuation-Dissipation ratio allow us to make the 


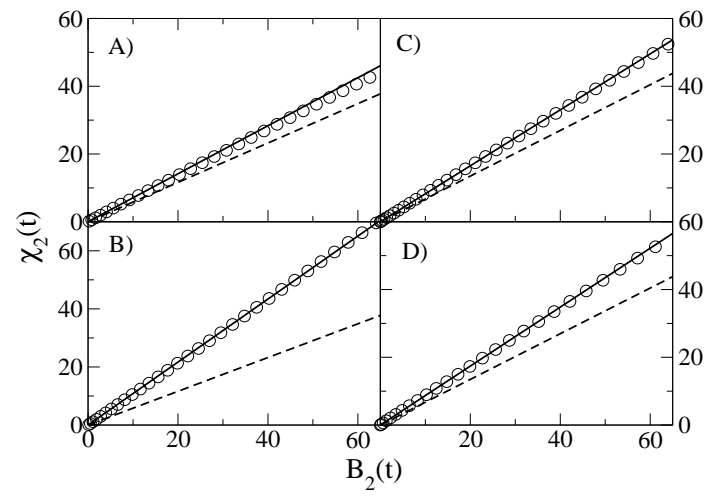

FIG. 6: Circles: Mobility vs. Diffusion of a single particle of mass $m_{\text {tracer }}$ in contact with $N=500$ particles of mass $m$, immersed in a heat bath (i.e. random kicks plus viscosity). We use the following conventions: $\alpha_{\text {tracer }}=\alpha_{12}$ and $\alpha=\alpha_{11}$. Only in case A) the tracer is also in contact with the external driving heat bath. A) $m_{\text {tracer }}=m, \alpha=0.9, \alpha_{\text {tracer }}=$ $\left.0.4, T_{g}=0.86, T_{g}^{\text {tracer }}=0.70 ; \mathbf{B}\right) m_{\text {tracer }}=m, \alpha=0.9$, $\left.\alpha_{\text {tracer }}=0.4, T_{g}=0.86, T_{g}^{\text {tracer }}=0.46 ; \mathbf{C}\right) m_{\text {tracer }}=7 \mathrm{~m}$, $\left.\alpha=\alpha_{\text {tracer }}=0.7, T_{g}=0.74, T_{g}^{\text {tracer }}=0.60 ; \mathbf{D}\right) m_{\text {tracer }}=$ $4 m, \alpha=\alpha_{\text {tracer }}=0.7, T_{g}=0.74, T_{g}^{\text {tracer }}=0.57$. The solid line has slope $1 /\left(2 T_{g}^{\text {tracer }}\right)$, the dashed line has slope $1 /\left(2 T_{g}\right)$.

following prediction. Suppose to perform a global measurement of the Fluctuation-Dissipation ratio on a system of unknown composition. A global measurement on a monodisperse system would yield a well defined FD ratio and a well defined temperature equal to the granular temperature, independently of the observable used (see [14]). On the other hand a polydisperse system would feature a typically non-constant FD ratio, function also of the observable (unless the system is completely elastic).

\section{The single tracer case}

Finally we investigate the special case $N_{2}=1$, i.e. the case of a single tracer, immersed in a granular gas of $N=N_{1}$ different particles, acting as a non-perturbing thermometer. Figure 6 reports the corresponding results. It turns out that, due to the inelasticity of collisions [13], the tracer reaches a granular temperature different from that of the surrounding particles. Measuring a FD relation thus yields the granular temperature of the tracer but not that of the surrounding granular gas. The tracer is thus sensing a temperature whose value is the outcome of the complex interaction between the tracer itself and the granular gas. It is worth stressing how this happens even if the tracer almost does not perturb the granular. This leads to the non-conventional result that the "temperature" measured as a fluctuation-dissipation ratio [15], in a driven granular gas, depends on the thermometer.

\section{CONCLUSIONS}

In this paper, we have shown by numerical simulations that, in a binary granular gas, each component of the mixture obeys a FD relation with its own granular temperature, while a global measurement provides a suitable definition of temperature only when equipartition is satisfied. These FD relations can be measured by different correlation-response pairs. In particular, the case of a tracer, which can act as a "thermometer" since it does not perturb the granular, has been investigated, and leads to the unusual conclusion that the measure of the temperature through FD relations would depend on the interaction between the tracer and the granular gas. Notice that theoretical studies (see e.g. 7]) may then allow for an estimate of the gas temperature, knowing the temperature measured by the tracer. Further investigations are needed to explore the scenarios bringing to violations of the FD relations [15]. This point becomes particularly important when, with more realistic energy injection through boundaries, we could expect heterogeneities giving rise to position-dependent temperatures. It would be interesting to investigate how a tracer particle would sample this inhomogeneous gas and ask about the meaning of the corresponding measured temperature.

Acknowledgments We are grateful to A. Baldassarri, H. J. Herrmann and E. Trizac for many enlightening discussions.

[1] T. Pöschel and S. Luding (Eds.), Granular Gases, Springer, Berlin (2001).

[2] Y. Limon Duparcmeur, Thèse de l'université de Rennes I (1996).

[3] W. Losert, D.G.W. Cooper, J. Delour, A. Kudrolli and J.P. Gollub, Chaos 9, 682 (1999).

[4] K. Feitosa and N. Menon, Phys. Rev. Lett. 88, 198301 (2002).

[5] R.D. Wildman and D.J. Parker, Phys. Rev. Lett. 88, 064301 (2002).

[6] V. Garzó and J. Dufty, Phys. Rev. E 605706 (1999).

[7] A. Barrat and E. Trizac, Gran. Matter 4, 57 (2002).

[8] J. M. Montanero and V. Garzó, Gran. Matter 4, 17 (2002).

[9] R. Clelland and C. M. Hrenya, Phys. Rev. E 65, 031301 (2002).

[10] U. Marini Bettolo Marconi and A. Puglisi, Phys. Rev. E 65, 051305 (2002) and Phys. Rev. E 66, 011301 (2002).

[11] R. Pagnani, U.M. Bettolo Marconi and A. Puglisi, Phys. Rev. E 66, 051304 (2002).

[12] A. Barrat and E. Trizac, Phys. Rev. E 66, 051303 (2002).

[13] Ph. A. Martin and J. Piasecki, Europhys. Lett. 46, 613 (1999).

[14] A. Puglisi, A. Baldassarri, and V. Loreto Phys. Rev. E 66, 061305 (2002).

[15] There exist in fact small, but systematic, deviations from FD relations even at small inelasticities. These deviations 
appear even at the homogeneous Boltzmann level (investigated by DSMC), where they are linked to the nonGaussian behaviour of the velocity distribution due to the departure from equilibrium [16]. The departure from (1) will be studied in a separate publication.

[16] J. Dufty and V. Garzò, J. Stat. Phys. 105, 723 (2001).

[17] G. D'Anna, P. Mayor, A. Barrat, V. Loreto and F. Nori, Nature 424, 909 (2003).

[18] F. Rouyer and N. Menon, Phys. Rev. Lett. 85, 3676 (2000).

[19] D.R.M. Williams and F.C. MacKintosh, Phys. Rev E 54, R9 (1996).

[20] A. Puglisi, V. Loreto, U. Marini Bettolo Marconi, A. Petri and A. Vulpiani, Phys. Rev. Lett 81, 3848 (1998); A. Puglisi, V. Loreto, U. Marini Bettolo Marconi and A. Vulpiani, Phys. Rev. E 59, 5582 (1999).

[21] T.P.C. van Noije and M.H. Ernst, Gran. Matter 1, 57 (1998).

[22] T.P.C. van Noije, M.H. Ernst, E. Trizac and I. Pagonabarraga, Phys. Rev. E 59, 4326 (1999); I. Pagonabarraga, E. Trizac, T.P.C. van Noije, and M.H. Ernst, Phys. Rev. E 65, 011303 (2002).

[23] J.M. Montanero and A. Santos, Granular Matter 2, 53
(2000).

[24] R. Cafiero, S. Luding and H.J. Herrmann, Phys. Rev. Lett. 84, 6014 (2000).

[25] S.J. Moon, M.D. Shattuck and J.B. Swift, Phys. Rev. E 64, 031303 (2001).

[26] It is also possible to use a deterministic thermostat. See e.g.: D. J. Evans, G. P. Morriss, Statistical Mechanics of Nonequilibrium Liquids, (Academic Press, London, 1990).

[27] We have checked the linearity of the response by changing the amplitude of the perturbation.

[28] G. Bird, Molecular Gas Dynamics, (Oxford University Press, New York, 1976) and Molecular Gas Dynamics and the Direct Simulation of Gas flows, (Clarendon Press, Oxford, 1994).

[29] M.S. Müller, Fast algorithms for the simulation of granukar particles, Ph.D. Thesis, Univ. of Stuttgart (2001); M.S. Müller and H.J. Herrmann, in Physics of Granular Media, H.J. Herrmann, J.-P. Hovi and S. Luding, NATO ASI Series, Kluwer Academic Publisher, Dordrecht (1998). 